\title{
Torus knots and quantum modular forms
}

\author{
Kazuhiro Hikami ${ }^{1 *}$ and Jeremy Lovejoy ${ }^{2 *}$
}

*Correspondence:

lovejoy@math.cnrs.fr

${ }^{1}$ Faculty of Mathematics, Kyushu University, Fukuoka 819-0395, Japan

${ }^{2}$ CNRS, LIAFA, Universite Denis Diderot - Paris 7, Case 7014, 75205

Paris Cedex 13, France khikami@gmail.com;

\begin{abstract}
In this paper we compute a $q$-hypergeometric expression for the cyclotomic expansion of the colored Jones polynomial for the left-handed torus knot $(2,2 t+1)$. We use this to define a family of $q$-series, the simplest case of which is the generating function for strongly unimodal sequences. Special cases of these $q$-series are quantum modular forms, and at roots of unity, these are dual to the generalized Kontsevich-Zagier series introduced by the first author. This duality generalizes a result of Bryson, Pitman, Ono, and Rhoades. We also compute Hecke-type expansions for our family of $q$-series.
\end{abstract}

2010 MSC: 11F37; 33D15; 57M27

Keywords: Torus knots; Colored Jones polynomial; Bailey pairs; Quantum modular forms

\section{Introduction and statement of results}

Several years ago, Zagier introduced quantum modular forms [30]. These are functions defined for $z \in \mathbb{Q}$ (or equivalently, for $q$ a root of unity, where $q:=e^{2 \pi i z}$ ) which behave like modular forms. For $g(z)$ to be quantum modular means that instead of requiring that $g(z)-\chi(\gamma)(c z+d)^{-k} g\left(\frac{a z+b}{c z+d}\right)=0$ for $\gamma=\left(\begin{array}{ll}a & b \\ c & d\end{array}\right) \in S L_{2}(\mathbb{Z})$ as with classical modular forms, we only ask that $g(z)-\chi(\gamma)(c z+d)^{-k} g\left(\frac{a z+b}{c z+d}\right)$ have 'nice' properties such as continuity or analyticity. The word quantum refers to the fact that these functions have 'the 'feel' of the objects in perturbative quantum field theory' (p. 659 of [30]). A celebrated example is the Kontsevich-Zagier series [29]

$$
F(q):=\sum_{n=0}^{\infty}(q)_{n}
$$

where we use the standard notation,

$$
(a)_{n}:=(1-a)(1-a q) \cdots\left(1-a q^{n-1}\right) .
$$

Note that $F(q)$ does not converge on any open subset of $\mathbb{C}$, but it is well-defined at any root of unity.

Bryson et al. [9] recently established a relationship between $F(q)$ and the generating function for strongly unimodal sequences,

$$
U(x ; q):=\sum_{n=0}^{\infty}(-x q)_{n}\left(-x^{-1} q\right)_{n} q^{n+1}
$$

(c) 2015 Hikami and Lovejoy; licensee Springer. This is an Open Access article distributed under the terms of the Creative 
Precisely, $U(-1 ; q)$ is dual to $F(q)$ at any root of unity $\zeta_{N}=\mathrm{e}^{2 \pi \mathrm{i} / N}$,

$$
F\left(\zeta_{N}^{-1}\right)=U\left(-1 ; \zeta_{N}\right)
$$

Note that, contrary to the Kontsevich-Zagier series $F(q)$, the function $U(x ; q)$ converges for generic $|q|<1$. It should be remarked that the function $U(-1 ; q)$ also plays a crucial role in studying a radial limit of a mock theta function of Ramanujan [11].

For other recent studies and interesting examples of quantum modular forms in number theory, combinatorics, knot theory, and Lie algebras, see e.g., [6-8,10,11,17,19,26,30].

This article is based on the observation that the identity (1.4) can be interpreted in terms of quantum topology as follows. We use the $N$-colored Jones polynomial $J_{N}(K ; q)$ for a knot $K$, which is based on the $N$-dimensional representation of $U_{q}\left(s \ell_{2}\right)$ (see e.g., [21]). Throughout this article, we use a normalization $J_{N}($ unknot; $q)=1$. It is known that

$$
J_{N}\left(K ; q^{-1}\right)=J_{N}\left(K^{*} ; q\right),
$$

where $K^{*}$ is a mirror image of $K$. The colored Jones polynomial for the right-handed trefoil $T_{(2,3)}$ and the left-handed trefoil $T_{(2,3)}^{*}$ may be, respectively, written as (see e.g., [12,20,24])

$$
\begin{aligned}
& J_{N}\left(T_{(2,3)} ; q\right)=q^{1-N} \sum_{n=0}^{\infty} q^{-n N}\left(q^{1-N}\right)_{n}, \\
& J_{N}\left(T_{(2,3)}^{*} ; q\right)=\sum_{n=0}^{\infty} q^{n}\left(q^{1-N}\right)_{n}\left(q^{1+N}\right)_{n} .
\end{aligned}
$$

(Note that the series defining $J_{N}\left(T_{(2,3)} ; q\right)$ and $J_{N}\left(T_{(2,3)}^{*} ; q\right)$ are in fact finite sums, terminating at $n=N)$. Letting $q=\zeta_{N}$ in (1.7) and $q=\zeta_{N}^{-1}$ in (1.6) and appealing to (1.5), one finds the duality in (1.4).

Based on the colored Jones polynomial for the torus knot $T_{(2,2 t+1)}$ at roots of unity, the first author [14] introduced a family of quantum modular forms generalizing $F(q)(1.1)$,

$$
F_{t}(q):=q^{t} \sum_{k_{t} \geq \cdots \geq k_{1} \geq 0}^{\infty}(q)_{k_{t}} \prod_{i=1}^{t-1} q^{k_{i}\left(k_{i}+1\right)}\left[\begin{array}{c}
k_{i+1} \\
k_{i}
\end{array}\right]_{q} .
$$

Here $\left[\begin{array}{l}n \\ k\end{array}\right]_{q}$ is the usual $q$-binomial coefficient,

$$
\left[\begin{array}{l}
n \\
k
\end{array}\right]_{q}:=\frac{(q)_{n}}{(q)_{n-k}(q)_{k}}
$$

Note that when $t=1$, we recover the Kontsevich-Zagier series, $F_{1}(q)=q F(q)$. Our purpose in this article is to use the perspective of quantum invariants to generalize $U(x ; q)$ and (1.4). As a dual to $F_{t}(q)$, we make the following definition.

Definition 1.1. The generalized $U$-function $U_{t}(x ; q)$ is defined by

$$
U_{t}(x ; q):=q^{-t} \sum_{k_{t} \geq \cdots \geq k_{1} \geq 1}(-x q)_{k_{t}-1}\left(-x^{-1} q\right)_{k_{t}-1} q^{k_{t}} \prod_{i=1}^{t-1} q^{k_{i}^{2}}\left[\begin{array}{c}
k_{i+1}+k_{i}-i+2 \sum_{j=1}^{i-1} k_{j} \\
k_{i+1}-k_{i}
\end{array}\right]_{q} .
$$


This function is well-defined for $|q|<1$. When $x=-1$, it is also defined when $q$ is a root of unity. Our first main result is the following generalization of (1.4).

\section{Theorem 1.2.}

$$
F_{t}\left(\zeta_{N}^{-1}\right)=U_{t}\left(-1 ; \zeta_{N}\right)
$$

Our second main result is a Hecke-type expansion for $U_{t}(x ; q)$. (The case $t=1$ and $x=-1$ appears in [9].)

Theorem 1.3. We have

$$
\begin{aligned}
& U_{t}(-x ; q) \\
& =-q^{-t / 2-1 / 8} \frac{(x q)_{\infty}(q / x)_{\infty}}{(q)_{\infty}^{2}} \\
& \times\left(\sum_{\substack{r, s \geq 0 \\
r \neq s}}-\sum_{\substack{r, s<0 \\
(\bmod 2)}}\right) \frac{(-1)^{\frac{r-s-1}{2}} q^{\frac{1}{8} r^{2}+\frac{4 t+3}{4} r s+\frac{1}{8} s^{2}+\frac{2+t}{2} r+\frac{t}{2} s}}{1-x q^{\frac{r+s+1}{2}}} \\
& =-q^{-t / 2-1 / 8} \frac{(x q)_{\infty}(q / x)_{\infty}}{(q)_{\infty}^{2}} \\
& \times\left(\sum_{\substack{r, s, u \geq 0 \\
r \neq s(\bmod 2)}}+\sum_{\substack{r, s, u<0 \\
r \neq s}}\right)(-1)^{\frac{r-s-1}{2}} x^{u} q^{\frac{1}{8} r^{2}+\frac{4 t+3}{4} r s+\frac{1}{8} s^{2}+\frac{2+t}{2} r+\frac{t}{2} s+u \frac{r+s+1}{2}} .
\end{aligned}
$$

The paper is constructed as follows. In the section 'Bailey pairs and the colored Jones polynomial', we review Bailey pairs and their relation to the colored Jones polynomial. In the section 'The colored Jones polynomial for torus knots', we study the colored Jones polynomial for the torus knot $T_{(2,2 t+1)}$. In particular, we use the Bailey pair machinery to compute the coefficients of the cyclotomic expansion of $J_{N}\left(T_{(2,2 t+1)}^{*} ; q\right)$, which leads to Theorem 1.2. In the section 'Hecke-type formulae', we prove Theorem 1.3, again by using the Bailey machinery. In the section 'The vector-valued case', we extend Theorems 1.2 and 1.3 to the vector-valued setting. We close with some suggestions for future research and an appendix containing some examples.

\section{Bailey pairs and the colored Jones polynomial}

In this section, we review facts about Bailey pairs and their relation to the colored Jones polynomial.

First recall [2] that two sequences $\left(\alpha_{n}, \beta_{n}\right)$ form a Bailey pair relative to $a$ if

$$
\beta_{n}=\sum_{j=0}^{n} \frac{\alpha_{j}}{(q)_{n-j}(a q)_{n+j}}
$$

or equivalently,

$$
\alpha_{n}=\frac{1-a q^{2 n}}{1-a} \frac{(a)_{n}}{(q)_{n}}(-1)^{n} q^{n(n-1) / 2} \sum_{j=0}^{n}\left(q^{-n}\right)_{j}\left(a q^{n}\right)_{j} q^{j} \beta_{j}
$$


The Bailey lemma [2] states that if $\left(\alpha_{n}, \beta_{n}\right)$ is a Bailey pair relative to $a$ then so is $\left(\alpha_{n}^{\prime}, \beta_{n}^{\prime}\right)$, where

$$
\alpha_{n}^{\prime}=\frac{(b)_{n}(c)_{n}(a q / b c)^{n}}{(a q / b)_{n}(a q / c)_{n}} \alpha_{n}
$$

and

$$
\beta_{n}^{\prime}=\sum_{k=0}^{n} \frac{(b)_{k}(c)_{k}(a q / b c)_{n-k}(a q / b c)^{k}}{(a q / b)_{n}(a q / c)_{n}(q)_{n-k}} \beta_{k} .
$$

In particular, if $b, c \rightarrow \infty$ then we have

$$
\alpha_{n}^{\prime}=a^{n} q^{n^{2}} \alpha_{n}
$$

and

$$
\beta_{n}^{\prime}=\sum_{k=0}^{n} \frac{a^{k} q^{k^{2}}}{(q)_{n-k}} \beta_{k}
$$

Inserting (2.3) and (2.4) back in Definition (2.1) and letting $n \rightarrow \infty$, we have

$$
\sum_{n \geq 0}(b)_{n}(c)_{n}(a q / b c)^{n} \beta_{n}=\frac{(a q / b)_{\infty}(a q / c)_{\infty}}{(a q)_{\infty}(a q / b c)_{\infty}} \sum_{n \geq 0} \frac{(b)_{n}(c)_{n}(a q / b c)^{n}}{(a q / b)_{n}(a q / c)_{n}} \alpha_{n} .
$$

Next, recall the cyclotomic expansion of the colored Jones polynomial due to Habiro [13]

$$
J_{N}(K ; q)=\sum_{n=0}^{\infty} C_{n}(K ; q)\left(q^{1+N}\right)_{n}\left(q^{1-N}\right)_{n},
$$

where we have

$$
C_{n}(K ; q) \in \mathbb{Z}\left[q, q^{-1}\right]
$$

The colored Jones polynomial $J_{N}(K ; q)$ and the coefficients $C_{n}(K ; q)$ defined in $(2.8)$ can be regarded as a Bailey pair $\left(\alpha_{n}, \beta_{n}\right)$ relative to $q^{2}$. Namely, comparing Equations (2.8) and (2.2) we have (see also $[13,16])$

$$
\begin{aligned}
& \alpha_{n}=\frac{\left(1-q^{n+1}\right)\left(1-q^{2 n+2}\right)}{(1-q)\left(1-q^{2}\right)}(-1)^{n} q^{\frac{1}{2} n(n-1)} J_{n+1}(K ; q), \\
& \beta_{n}=q^{-n} C_{n}(K ; q) .
\end{aligned}
$$

Equation (2.1) gives the inverse transform

$$
C_{n}(K ; q)=-q^{n+1} \sum_{\ell=1}^{n+1} \frac{\left(1-q^{\ell}\right)\left(1-q^{2 \ell}\right)}{(q)_{n+1-\ell}(q)_{n+1+\ell}}(-1)^{\ell} q^{\frac{1}{2} \ell(\ell-3)} J_{\ell}(K ; q) .
$$

\section{The colored Jones polynomial for torus knots}

For some knots $K$, explicit forms of $J_{N}(K ; q)$ and/or $C_{N}(K ; q)$ are known in the literature. For instance, when $K$ is the right-handed torus knot $T_{(s, t)}$, where $s$ and $t$ are coprime positive integers, the colored Jones polynomial is given by $[25,27]$

$$
J_{N}\left(T_{(s, t)} ; q\right)=\frac{q^{\frac{1}{4} s t\left(1-N^{2}\right)}}{q^{\frac{N}{2}}-q^{-\frac{N}{2}}} \sum_{j=-\frac{N-1}{2}}^{\frac{N-1}{2}} q^{s t j^{2}}\left(q^{-(s+t) j+\frac{1}{2}}-q^{-(s-t) j-\frac{1}{2}}\right) .
$$


Using difference equations, the first author [14] constructed a $q$-hypergeometric expression for $J_{N}\left(T_{(s, t)} ; q\right)$ when $s=2$,

$$
J_{N}\left(T_{(2,2 t+1)} ; q\right)=q^{t(1-N)} \sum_{k_{t} \geq \cdots \geq k_{1} \geq 0}^{\infty}\left(q^{1-N}\right)_{k_{t}} q^{-N k_{t}} \prod_{i=1}^{t-1} q^{k_{i}\left(k_{i}+1-2 N\right)}\left[\begin{array}{c}
k_{i+1} \\
k_{i}
\end{array}\right]_{q} .
$$

(See [18] for similar expressions for some other torus knots.) Comparing this with the generalized Kontsevich-Zagier series $(1.8)$, we find that $J_{N}\left(T_{(2,2 t+1)} ; q\right)$ and $F_{t}(q)$ agree at roots of unity,

$$
J_{N}\left(T_{(2,2 t+1)} \zeta_{N}\right)=F_{t}\left(\zeta_{N}\right)
$$

With (1.5) and (2.8) in mind, we see that to discover Definition 1.1 and prove Theorem 1.2, we need to compute the cyclotomic expansion of the colored Jones polynomial of the left-handed torus $\operatorname{knot} T_{(2,2 t+1)}^{*}$. Recalling that the colored Jones polynomial for the mirror image $K^{*}$ is given from that for $K(1.5)$, we find from (3.1) that

$$
\left(1-q^{N}\right) J_{N}\left(T_{(2,2 t+1)}^{*} q\right)=(-1)^{N} q^{-t+\frac{1}{2} N+\frac{2 t+1}{2} N^{2}} \sum_{k=-N}^{N-1}(-1)^{k} q^{-\frac{2 t+1}{2} k(k+1)+k} .
$$

Then, the coefficients $C_{n}$ in the cyclotomic expansion (2.8) are given from the inverse transform (2.11) as

$$
\begin{aligned}
& C_{n-1}\left(T_{(2,2 t+1)}^{*} ; q\right) \\
& \quad=-q^{n-t} \sum_{\ell=0}^{n} \frac{1}{(q)_{n-\ell}(q)_{n+\ell}} q^{(t+1) \ell^{2}-\ell}\left(1-q^{2 \ell}\right) \sum_{k=-\ell}^{\ell-1}(-1)^{k} q^{-\left(t+\frac{1}{2}\right) k^{2}-\left(t-\frac{1}{2}\right) k} .
\end{aligned}
$$

In the following proposition, we give a $q$-hypergeometric expression for the coefficients $C_{n}\left(T_{(2,2 t+1)}^{*} q\right)$. We use the usual characteristic function

$$
\chi(X):= \begin{cases}1, & \text { when } X \text { is true } \\ 0, & \text { when } X \text { is false. }\end{cases}
$$

Proposition 3.1. We have

$$
\begin{aligned}
& -q^{t-n} C_{n-1}\left(T_{(2,2 t+1)}^{*} ; q\right) \\
& =\sum_{n \geq n_{2 t-1} \geq \cdots \geq n_{1} \geq 0} \frac{q^{\sum_{i=1}^{t-1} n_{t+i}^{2}+\left(\begin{array}{c}
n_{t} \\
2
\end{array}\right)-\sum_{i=1}^{t-1} n_{i} n_{i+1}-\sum_{i=1}^{t-2} n_{i}}(-1)^{n_{t}}\left(1-q^{n_{t}-\chi(t \geq 2) n_{t-1}}\right)}{(q)_{n-n_{2 t-1}}(q)_{n_{2 t-1}-n_{2 t-2}} \cdots(q)_{n_{2}-n_{1}}(q)_{n_{1}}} .
\end{aligned}
$$

Proof. In light of Equations (3.5) and (2.1), we need to find $\beta_{n}^{\prime}$ such that $\left(\alpha_{n}^{\prime}, \beta_{n}^{\prime}\right)$ form a Bailey pair relative to 1 , where

$$
\alpha_{n}^{\prime}=q^{(t+1) n^{2}-n}\left(1-q^{2 n}\right) \sum_{j=-n}^{n-1}(-1)^{j} q^{-\left((2 t+1) j^{2}+(2 t-1) j\right) / 2} .
$$


We require a result of the second author. Namely, in part (ii) of Theorem 1.1 of [23], let $k=K=t, \ell=t-1$, and $m=0$. Then, $\left(\alpha_{n}, \beta_{n}\right)$ form a Bailey pair relative to 1 , where

$$
\beta_{n}=\sum_{n \geq n_{2 t-1} \geq \cdots \geq n_{1} \geq 0} \frac{q^{\sum_{i=1}^{t-1} n_{t+i}^{2}+\left({ }^{n_{t}+1}{ }_{2}\right)-\sum_{i=1}^{t-1} n_{i} n_{i+1}-\sum_{i=1}^{t-1} n_{i}}(-1)^{n_{t}}}{(q)_{n-n_{2 t-1}}(q)_{n_{2 t-1}-n_{2 t-2}} \cdots(q)_{n_{2}-n_{1}}(q)_{n_{1}}}
$$

and

$$
\begin{aligned}
\alpha_{n}= & q^{(t+1) n^{2}+n} \sum_{j=-n}^{n}(-1)^{j} q^{-\left((2 t+1) j^{2}+(2 t-1) j\right) / 2} \\
& -\chi(n \neq 0) q^{(t+1) n^{2}-n} \sum_{j=-n+1}^{n-1}(-1)^{j} q^{-\left((2 t+1) j^{2}+(2 t-1) j\right) / 2} \\
= & -q^{(t+1) n^{2}-n}\left(1-q^{2 n}\right) \sum_{j=-n}^{n-1}(-1)^{j} q^{-\left((2 t+1) j^{2}+(2 t-1) j\right) / 2} \\
& + \begin{cases}1, & \text { if } n=0, \\
(-1)^{n} q^{\frac{n^{2}}{2}+\left(\frac{2 t-3}{2}\right) n}+(-1)^{n} q^{\frac{n^{2}}{2}-\left(\frac{2 t-3}{2}\right) n}, & \text { if } n \geq 1 .\end{cases}
\end{aligned}
$$

Let $\alpha_{n}=-\alpha_{n}^{\prime}+\alpha_{n}^{\prime \prime}=(3.9)+(3.10)$. To find $\beta_{n}^{\prime \prime}$, we start with Equations (3.9) and (3.10) of [23], which state that

$$
\alpha_{n}^{*}= \begin{cases}1, & \text { if } n=0, \\ (-1)^{n}\left(q^{\left(-(2 k-1) n^{2}-(2 \ell+1) n\right) / 2}+q^{\left(-(2 k-1) n^{2}+(2 \ell+1) n\right) / 2}\right), & \text { if } n>0,\end{cases}
$$

and

$$
\beta_{n}^{*}=\beta_{n_{k}}=(-1)^{n_{k}} q^{-\left(\begin{array}{c}
n_{k}+1 \\
2
\end{array}\right)} \sum_{n_{k} \geq n_{k-1} \geq \cdots \geq n_{1} \geq 0} \frac{q^{-\sum_{i=1}^{k-1} n_{i} n_{i+1}-\sum_{i=1}^{\ell} n_{i}}}{(q)_{n_{k}-n_{k-1}} \cdots(q)_{n_{2}-n_{1}}(q)_{n_{1}}},
$$

form a Bailey pair relative to 1 . Using this Bailey pair with $k=t$ and

$$
\ell= \begin{cases}0, & \text { for } t=1 \\ t-2, & \text { for } t \geq 2\end{cases}
$$

we iterate Equations (2.5) and (2.6) $t$ times. Then, $\alpha_{n}^{*}$ becomes $\alpha_{n}^{\prime \prime}$ and

$$
\beta_{n}^{\prime \prime}=\sum_{n \geq n_{2 t-1} \geq \cdots \geq n_{1} \geq 0} \frac{q^{\sum_{i=1}^{t-1} n_{t+i}^{2}+\left(\begin{array}{c}
n_{t} \\
2
\end{array}\right)-\sum_{i=1}^{t-1} n_{i} n_{i+1}-\sum_{i=1}^{t-2} n_{i}}(-1)^{n_{t}}}{(q)_{n-n_{2 t-1}}(q)_{n_{2 t-1}-n_{2 t-2}} \cdots(q)_{n_{2}-n_{1}}(q)_{n_{1}}} .
$$

Taking $\beta_{n}^{\prime \prime}-\beta_{n}$ gives the expression for $-q^{t-n} C_{n-1}\left(T_{(2,2 t+1)}^{*}\right)$.

While the above proposition does furnish an attractive $q$-hypergeometric expression for $C_{n}\left(T_{(2,2 t+1)}^{*} q\right)$, it is not apparent that these coefficients are Laurent polynomials in $q$, as guaranteed by (2.9). This is made clear with the next proposition. 
Proposition 3.2. We have

$$
\begin{aligned}
C_{n}\left(T_{(2,2 t+1)}^{*} ; q\right) & =q^{n+1-t} \sum_{n+1=k_{t} \geq k_{t-1} \geq \cdots \geq k_{1} \geq 1} \prod_{a=1}^{t-1} q^{k_{a}^{2}} \frac{\left(q^{1-a+\sum_{i=1}^{a} 2 k_{i}}\right)_{k_{a+1}-k_{a}}}{(q)_{k_{a+1}-k_{a}}} \\
& =q^{n+1-t} \sum_{n+1=k_{t} \geq k_{t-1} \geq \cdots \geq k_{1} \geq 1} \prod_{i=1}^{t-1} q^{k_{i}^{2}}\left[\begin{array}{c}
k_{i+1}+k_{i}-i+2 \sum_{j=1}^{i-1} k_{j} \\
k_{i+1}-k_{i}
\end{array}\right]_{q}
\end{aligned}
$$

Proof. We recall the classical $q$-binomial identity,

$$
\sum_{n=0}^{N} z^{n} q^{\left(\begin{array}{c}
n \\
2
\end{array}\right)}\left[\begin{array}{c}
N \\
n
\end{array}\right]_{q}=(z)_{N}
$$

Letting $z=-z q^{a}, N=b-a$, and shifting $n$ to $n-a$, we have the identity

$$
\sum_{n=a}^{b} \frac{q^{\left(\begin{array}{c}
n \\
2
\end{array}\right)}(-z)^{n}}{(q)_{b-n}(q)_{n-a}}=(-z)^{a} q^{\left(\begin{array}{c}
a \\
2
\end{array}\right)} \frac{\left(z q^{a}\right)_{b-a}}{(q)_{b-a}}
$$

Using this identity, we also have, for arbitrary $c$,

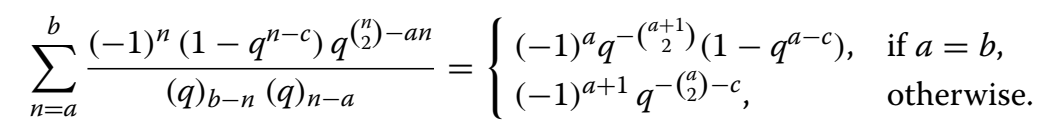

We may use the two identities (3.17) and (3.18) to transform (3.6) into (3.14) as follows. First, if $n_{t+1}=n_{t-1}$ then the sum in (3.6) vanishes, so we may assume $n_{t+1}>n_{t-1}$. The sum over $n_{t}$ is

$$
\sum_{n_{t}=n_{t-1}}^{n_{t+1}} \frac{(-1)^{n_{t}} q^{\left(\begin{array}{c}
n_{t} \\
2
\end{array}\right)-n_{t-1} n_{t}}\left(1-q^{n_{t}-n_{t-1}}\right)}{(q)_{n_{t+1}-n_{t}}(q)_{n_{t}-n_{t-1}}}
$$

and the second part of identity (3.18) then enables us to evaluate this sum, giving

$$
\begin{aligned}
-q^{t-n-1} C_{n}\left(T_{(2,2 t+1)}^{*} ; q\right) & \sum_{n+1 \geq n_{2 t-1} \geq \cdots \geq n_{t+2} \geq n_{t+1}>n_{t-1} \geq n_{t-2} \geq \cdots \geq n_{1} \geq 0}(-1)^{1+n_{t-1}} q^{\sum_{i=1}^{t-1} n_{t+i}^{2}-\sum_{i=1}^{t-2} n_{i} n_{i+1}-\sum_{i=1}^{t-1} n_{i}} \\
& \times \frac{q^{-\left(\begin{array}{c}
c_{t-1} \\
2
\end{array}\right)}}{(q)_{n+1-n_{2 t-1}} \cdots(q)_{n_{t+2}-n_{t+1}}(q)_{n_{t-1}-n_{t-2}} \cdots(q)_{n_{2}-n_{1}}(q)_{n_{1}}} .
\end{aligned}
$$

We then set $n_{t+1}=n_{t-1}+k_{1}$, with $k_{1} \geq 1$. The sum over $n_{t-1}$ is

$$
\sum_{n_{t-1}=n_{t-2}}^{n_{t+2}-k_{1}} \frac{(-1)^{n_{t-1}} q^{\left(\begin{array}{c}
n_{t-1} \\
2
\end{array}\right)-n_{t-2} n_{t-1}+2 k_{1} n_{t-1}}}{(q)_{n_{t+2}-k_{1}-n_{t-1}}(q)_{n_{t-1}-n_{t-2}}}
$$


and (3.17) allows us to evaluate this sum, resulting in

$$
\begin{aligned}
q^{t-n-1} C_{n}\left(T_{(2,2 t+1)}^{*} ; q\right) & \sum_{n+1 \geq n_{2 t-1} \geq \cdots \geq n_{t+2} \geq n_{t-2}+k_{1}>n_{t-2} \geq \cdots \geq n_{1} \geq 0}(-1)^{n_{t-2}} q^{\sum_{i=2}^{t-1} n_{t+i}^{2}-\sum_{i=1}^{t-3} n_{i} n_{i+1}-\sum_{i=1}^{t-2} n_{i}} \\
& \times \frac{\left(q^{2 k_{1}}\right)_{n_{t+2}-n_{t-2}-k_{1}}}{(q)_{n_{t+2}-n_{t-2}-k_{1}}} \cdot \frac{q^{\left.-{ }^{\left({ }^{t-2}\right.}{ }_{2}\right)-n_{t-2}+2 k_{1} n_{t-2}+k_{1}{ }^{2}}}{(q)_{n+1-n_{2 t-1}} \cdots(q)_{n_{t+3}-n_{t+2}}(q)_{n_{t-2}-n_{t-3}} \cdots(q)_{n_{2}-n_{1}}(q)_{n_{1}}} .
\end{aligned}
$$

We continue in the same manner, next setting $n_{t+2}=n_{t-2}+k_{2}$, with $k_{2} \geq k_{1}$, and we may then take the sum over $n_{t-2}$ using (3.17). Iterating this process (taking the sum over $n_{t-a}$ after setting $n_{t+a}=n_{t-a}+k_{a}$ ), we arrive at (3.14). The expression in Equation (3.15) follows from the fact that

$$
\left[\begin{array}{l}
n \\
k
\end{array}\right]_{q}=\frac{\left(q^{k+1}\right)_{n-k}}{(q)_{n-k}} .
$$

We are now prepared to prove the duality in Theorem 1.2.

Proof of Theorem 1.2. Comparing Equations (3.14) and (1.10), we see that

$$
U_{t}(x ; q)=\sum_{n=0}^{\infty} C_{n}\left(T_{(2,2 t+1)}^{*} ; q\right)(-x q)_{n}\left(-x^{-1} q\right)_{n} .
$$

Therefore, by $(2.8), U_{t}(x ; q)$ gives the colored Jones polynomial for $T_{(2,2 t+1)}^{*}$ when $x=q^{N}$,

$$
J_{N}\left(T_{(2,2 t+1)}^{*} ; q\right)=U_{t}\left(-q^{N} ; q\right) .
$$

Combining (3.22) with (3.3) and (1.5) gives the statement of the theorem.

\section{Hecke-type formulae}

In this section, we prove Theorem 1.3 as well as a simpler formula when $t=1$.

Proof of Theorem 1.3. In Equations (2.5) and (2.6), we let $a=1, b=1 / c=x$ and apply $\alpha_{n}^{\prime}$ and $\beta_{n}^{\prime}=-q^{t-n} C_{n-1}$ from the proof of Proposition 3.1. Recalling (3.21), we obtain

$$
\begin{aligned}
U_{t}(-x ; q)= & \frac{-q^{-t}(x q)_{\infty}(q / x)_{\infty}}{(q)_{\infty}^{2}} \sum_{n \geq 1} \sum_{k=-n}^{n-1} \frac{(-1)^{k} q^{(t+1) n^{2}-\left(t+\frac{1}{2}\right) k^{2}-\left(t-\frac{1}{2}\right) k}\left(1-q^{2 n}\right)}{\left(1-x q^{n}\right)\left(1-q^{n} / x\right)} \\
= & \frac{-q^{-t}(x q)_{\infty}(q / x)_{\infty}}{(q)_{\infty}^{2}} \sum_{n \geq 1} \sum_{k=-n}^{n-1} \frac{(-1)^{k} q^{(t+1) n^{2}-\left(t+\frac{1}{2}\right) k^{2}-\left(t-\frac{1}{2}\right) k}}{1-x q^{n}} \\
& +\frac{-q^{-t}(x q)_{\infty}(q / x)_{\infty}}{(q)_{\infty}^{2}} \sum_{n \geq 1} \sum_{k=-n}^{n-1} \frac{(-1)^{k} q^{(t+1) n^{2}+n-\left(t+\frac{1}{2}\right) k^{2}-\left(t-\frac{1}{2}\right) k}}{x\left(1-q^{n} / x\right)} \\
= & \frac{-q^{-t}(x q)_{\infty}(q / x)_{\infty}}{(q)_{\infty}^{2}} \sum_{n \geq 1} \sum_{k=-n}^{n-1} \frac{(-1)^{k} q^{(t+1) n^{2}-\left(t+\frac{1}{2}\right) k^{2}-\left(t-\frac{1}{2}\right) k}}{1-x q^{n}} \\
& -\frac{-q^{-t}(x q)_{\infty}(q / x)_{\infty}}{(q)_{\infty}^{2}} \sum_{n \leq 1} \sum_{k=n}^{-n-1} \frac{(-1)^{k} q^{(t+1) n^{2}-\left(t+\frac{1}{2}\right) k^{2}-\left(t-\frac{1}{2}\right) k}}{1-x q^{n}} .
\end{aligned}
$$


Letting $n=(r+s+1) / 2$ and $k=(r-s-1) / 2$ in each of the two final sums leads to the expression in (1.12). The expression in (1.13) follows upon expanding the term $1 /\left(1-x q^{(r+s+1) / 2}\right)$ in (1.12) as a geometric series.

When $t=1$, we have the following Hecke-type double sum.

\section{Theorem 4.1.}

$$
(1-x) U_{1}(-x ; q)=\frac{1}{(q)_{\infty}}\left(\sum_{r, n \geq 0}-\sum_{r, n<0}\right)(-1)^{n+r} x^{-r} q^{n(3 n+5) / 2+2 n r+r(r+3) / 2} .
$$

Proof. To see this, we use the Bailey pair relative to $q$ (Lemma 6 of [3]),

$$
\alpha_{n}=(-x)^{-n} q^{\left(\begin{array}{c}
n+1 \\
2
\end{array}\right)}\left(1-x^{2 n+1}\right)
$$

and

$$
\beta_{n}=\frac{(x)_{n+1}(q / x)_{n}}{\left(q^{2}\right)_{2 n}}
$$

together with the fact that if $\left(\alpha_{n}, \beta_{n}\right)$ is a Bailey pair relative to $a$, then by Corollary 1.3 of [22] we have

$$
\sum_{n \geq 0}(a q)_{2 n} q^{n} \beta_{n}=\frac{1}{(q)_{\infty}} \sum_{r, n \geq 0}(-a)^{n} q^{3 n(n+1) / 2+(2 n+1) r} \alpha_{r} .
$$

This gives

$$
\sum_{n \geq 0}(x)_{n+1}(q / x)_{n} q^{n}=\frac{1}{(q)_{\infty}} \sum_{r, n \geq 0}(-1)^{n+r} x^{-r} q^{n(3 n+5) / 2+2 n r+r(r+3) / 2}\left(1-x^{2 r+1}\right) .
$$

Using $\left(1-x^{2 r+1}\right)$ to split the right-hand side into two sums and then replacing $(r, n)$ by $(-r-1,-n-1)$ in the second sum yields the result.

\section{The vector-valued case}

The first author [15] introduced and studied the modular properties of a family of $q$-series $F_{t}^{(m)}(q)$, defined for $1 \leq m \leq t$ by

$$
F_{t}^{(m)}(q):=q^{t} \sum_{k_{1}, \ldots, k_{t}=0}^{\infty}(q)_{k_{t}} q^{k_{1}^{2}+\cdots+k_{t-1}^{2}+k_{m}+\cdots+k_{t-1}} \prod_{i=1}^{t-1}\left[\begin{array}{c}
k_{i+1}+\delta_{i, m-1} \\
k_{i}
\end{array}\right]_{q} .
$$

The case $m=1$ corresponds to (1.8), $F_{t}(q)=F_{t}^{(1)}(q)$.

He showed [15] that at the $N$-th root of unity we have

$$
\zeta_{N}^{-t+\frac{(2 t+1-2 m)^{2}}{8(2 t+1)}} F_{t}^{(m)}\left(\zeta_{N}\right)=(2 t+1) N \sum_{k=1}^{4(2 t+1) N} \chi_{8 t+4}^{(m)}(k) \zeta_{N}^{\frac{k^{2}}{8(2 t+1)}} B_{2}\left(\frac{k}{4(2 t+1) N}\right)
$$

where the Bernoulli polynomial is $B_{2}(x)=x^{2}-x+\frac{1}{6}$, and the periodic function is

$$
\chi_{8 t+4}^{(m)}(k):=\left\{\begin{array}{lll}
1, & \text { when } k= \pm(2 t+1-2 m) & \bmod 8 t+4, \\
-1, & \text { when } k= \pm(2 t+1+2 m) & \bmod 8 t+4, \\
0, & \text { otherwise. }
\end{array}\right.
$$


This is a limiting value of the Eichler integral of a vector-valued modular form $\Phi_{t}^{(m)}(\tau)$ with weight $1 / 2$,

$$
\begin{aligned}
\Phi_{t}^{(m)}(\tau) & :=\sum_{n=0}^{\infty} \chi_{8 t+4}^{(m)}(n) q^{\frac{1}{8(2 t+1)} n^{2}} \\
& =q^{\frac{(2 t+1-2 m)^{2}}{8(2 t+1)}}\left(q^{m}, q^{2 t+1-m}, q^{2 t+1} ; q^{2 t+1}\right)_{\infty},
\end{aligned}
$$

where as usual $q=\mathrm{e}^{2 \pi \mathrm{i} \tau}$ and $(a, b, \cdots ; q)_{\infty}=(a)_{\infty}(b)_{\infty} \cdots$. Note that these $q$-series appeared in the Andrews-Gordon identities [1], the $t=2$ case of which corresponds to the Rogers-Ramanujan identities. The quantum modularity of $F_{t}^{(m)}(q)$ is given by

$$
\begin{array}{r}
\phi_{t}^{(m)}(z)+\frac{1}{(\mathrm{i} z)^{\frac{3}{2}}} \sum_{m^{\prime}=1}^{t} \frac{2}{\sqrt{2 t+1}}(-1)^{t+1+m+m^{\prime}} \sin \left(\frac{2 m m^{\prime}}{2 t+1} \pi\right) \phi_{t}^{\left(m^{\prime}\right)}(-1 / z) \\
=\frac{\sqrt{(2 t+1) \mathrm{i}}}{2 \pi} \int_{0}^{\mathrm{i} \infty} \frac{\Phi_{t}^{(m)}(w)}{(w-z)^{\frac{3}{2}}} d w,
\end{array}
$$

where $z \in \mathbb{Q}$ and $\phi_{t}^{(m)}(\tau):=q^{-t+\frac{(2 t+1-2 m)^{2}}{8(2 t+1)}} F_{t}^{(m)}(q)$. See [15] for details.

In this section, we define $q$-series $U_{t}^{(m)}(q)$ so that $F_{t}^{(m)}\left(\zeta_{N}^{-1}\right)=U_{t}^{(m)}\left(-1 ; \zeta_{N}\right)$ (see Theorem 5.5) and we find the Hecke-type formulae for $U_{t}^{(m)}(q)$ (see Theorem 5.6). We begin by defining an analogue of the colored Jones polynomial,

$$
\left(1-q^{N}\right) J_{N}^{(t, m)}(q):=(-1)^{N} q^{-t+\frac{N}{2}+\frac{2 t+1}{2} N^{2}} \sum_{k=-N}^{N-1}(-1)^{k} q^{-\frac{2 t+1}{2} k(k+1)+m k} .
$$

When $m=1$, this coincides with the colored Jones polynomial $J_{N}^{(t, 1)}(q)=$ $J_{N}\left(T_{(2,2 t+1)}^{*} ; q\right)$.

\section{Proposition 5.1.}

$$
J_{N}^{(t, m)}\left(\zeta_{N}\right)=F_{t}^{(m)}\left(\zeta_{N}^{-1}\right)
$$

Proof. The function $J_{N}^{(t, m)}(q)$ is also written by use of the periodic function (5.3) as

$$
\left(1-q^{N}\right) J_{N}^{(t, m)}(q)=(-1)^{N} q^{-t+\frac{1}{2} N+\frac{2 t+1}{2} N^{2}} \sum_{k=1}^{2(2 t+1) N} \chi_{8 t+4}^{(m)}(k) q^{-\frac{k^{2}-(2 t+1-2 m)^{2}}{8(2 t+1)}} .
$$

At $q \rightarrow \zeta_{N}$, we have

$$
\begin{aligned}
\zeta_{N}^{t-\frac{(2 t+1-2 m)^{2}}{8(2 t+1)}} J_{N}^{(t, m)}\left(\zeta_{N}\right) & =-\lim _{q \rightarrow \zeta_{N}} \frac{1}{1-q^{N}} \sum_{k=1}^{2(2 t+1) N} \chi_{8 t+4}^{(m)}(k) q^{-\frac{k^{2}}{8(2 t+1)}} \\
& =-\frac{1}{8(2 t+1) N} \sum_{k=1}^{2(2 t+1) N} k^{2} \chi_{8 t+4}^{(m)}(k) \zeta_{N}^{-\frac{k^{2}}{8(2 t+1)}} .
\end{aligned}
$$

Using (5.3), the sum $\sum_{k=0}^{2(2 t+1) N}$ may be replaced with $\frac{1}{2} \sum_{k=-2(2 t+1) N}^{2(2 t+1) N}$. Then the righthand side is written as

$$
-\frac{1}{16(2 t+1) N} \sum_{\ell=0}^{4(2 t+1) N}(\ell-2(2 t+1) N)^{2} \chi_{8 t+4}^{(m)}(\ell-2(2 t+1) N) \zeta_{N}^{-\frac{(\ell-2(2 t+1) N)^{2}}{8(2 t+1)}} .
$$


Since $\chi_{8 t+4}^{(m)}(k-2(2 t+1))=-\chi_{8 t+4}^{(m)}(k)$, we obtain

$$
\zeta_{N}^{t-\frac{(2 t+1-2 m)^{2}}{8(2 t+1)}} J_{N}^{(t, m)}\left(\zeta_{N}\right)=(2 t+1) N \sum_{k=1}^{4(2 t+1) N} \chi_{8 t+4}^{(m)}(k) \zeta_{N}^{-\frac{k^{2}}{8(2 t+1)}} B_{2}\left(\frac{k}{4(2 t+1) N}\right) .
$$

Recalling (5.2), the statement follows.

Next, we study a cyclotomic expansion for $J_{N}^{(t, m)}(q)$,

$$
J_{N}^{(t, m)}(q)=\sum_{n=0}^{\infty} C_{n}^{(t, m)}(q)\left(q^{1+N}\right)_{n}\left(q^{1-N}\right)_{n}
$$

Equation (2.11) shows that we have

$$
C_{n-1}^{(t, m)}(q)=-q^{n-t} \sum_{\ell=0}^{n} \frac{1}{(q)_{n-\ell}(q)_{n+\ell}} q^{(t+1) \ell^{2}-\ell}\left(1-q^{2 \ell}\right) \sum_{k=-\ell}^{\ell-1}(-1)^{k} q^{-\frac{2 t+1}{2} k(k+1)+m k} .
$$

We note that $C_{n}^{(t, 1)}(q)=C_{n}\left(T_{(2,2 t+1)}^{*} ; q\right)$.

The next two propositions are generalizations of Propositions 3.1 and 3.2, respectively.

Proposition 5.2. We have

$$
-q^{t-n} C_{n-1}^{(t, m)}=\sum_{n \geq n_{2 t-1} \geq \cdots \geq n_{1} \geq 0} \frac{q^{\sum_{i=1}^{t-1} n_{t+i}^{2}+\left(\begin{array}{c}
n_{t} \\
2
\end{array}\right)-\sum_{i=1}^{t-1} n_{i} n_{i+1}-\sum_{i=1}^{t-m-1} n_{i}}(-1)^{n_{t}}\left(1-q^{n_{t}-\chi(t>m) n_{t-m}}\right)}{(q)_{n-n_{2 t-1}} \cdots(q)_{n_{2}-n_{1}}(q)_{n_{1}}} .
$$

Proof. In light of Equation (5.10) and the definition of a Bailey pair (2.1), we need to find $\beta_{n}^{\prime}$ such that

$$
\alpha_{n}^{\prime}=q^{(t+1) n^{2}-n}\left(1-q^{2 n}\right) \sum_{k=-n}^{n-1}(-1)^{k} q^{-(2 t+1) k^{2} / 2-(2 t-(2 m-1)) k / 2} .
$$

The proof of this Bailey pair is exactly as in the proof of Proposition 3.1 but with $\ell=$ $t-m$ instead of $t-1$.

Proposition 5.3. We have

$$
\begin{aligned}
C_{n}^{(t, m)}(q) & =q^{n+1-t} \sum_{\substack{n+1=k_{t} \geq k_{t-1} \geq \cdots \geq k_{1} \geq 0 \\
k_{m} \geq 1}} \prod_{a=1}^{t-1} q^{k_{a}^{2}} \frac{\left(q^{1-a+\sum_{i=1}^{a}\left(2 k_{i}+\chi(m>i)\right)}\right)_{k_{a+1}-k_{a}}}{(q)_{k_{a+1}-k_{a}}} \\
& =q^{n+1-t} \sum_{n+1=k_{t} \geq k_{t-1} \geq \cdots \geq k_{1} \geq 0} \prod_{i=1}^{t-1} q^{k_{i}^{2}}\left[\begin{array}{c}
k_{i+1}-k_{i}-i+\sum_{j=1}^{i}\left(2 k_{j}+\chi(m>j)\right) \\
k_{i+1}-k_{i}
\end{array}\right] .
\end{aligned}
$$


Proof. The proof is similar to that of Proposition 3.2. We begin by treating the sum over $n_{t}$, which is

$$
\sum_{n_{t}=n_{t-1}}^{n_{t+1}} \frac{(-1)^{n_{t}} q^{\left(\begin{array}{c}
n_{t} \\
2
\end{array}\right)-n_{t-1} n_{t}}\left(1-q^{n_{t}-\chi(t>m) n_{t-m}}\right)}{(q)_{n_{t+1}-n_{t}}(q)_{n_{t}-n_{t-1}}} .
$$

Assuming for the moment that $n_{t+1}>n_{t-1}$, the second part of identity (3.18) enables us to evaluate this sum, giving

$$
\begin{aligned}
& -q^{t-n-1} C_{n}^{(t, m)}(q) \\
& =\sum_{n+1 \geq n_{2 t-1} \geq \cdots \geq n_{t+2} \geq n_{t+1}>n_{t-1} \geq n_{t-2} \geq \cdots \geq n_{1} \geq 0}(-1)^{1+n_{t-1}} q^{\sum_{i=1}^{t-1} n_{t+i}^{2}-\sum_{i=1}^{t-2} n_{i} n_{i+1}-\sum_{i=1}^{t-m} n_{i}} \\
& \times \frac{q^{-\left(\begin{array}{c}
n_{t-1} \\
2
\end{array}\right)}}{(q)_{n+1-n_{2 t-1}} \cdots(q)_{n_{t+2}-n_{t+1}}(q)_{n_{t-1}-n_{t-2}} \cdots(q)_{n_{2}-n_{1}}(q)_{n_{1}}} \text {. }
\end{aligned}
$$

The rest of the proof is the same as the proof of Proposition 3.2. The only difference between Equations (3.19) and (5.16) is that the latter contains the term $q^{-\sum_{i=1}^{t-m} n_{i}}$ instead of the term $q^{-\sum_{i=1}^{t-1} n_{i}}$, which results in (5.13) instead of (3.14).

Now, suppose that $n_{t+1}=n_{t-1}$. The sum (5.15) on $n_{t}$ is trivial and reduces to

$$
(-1)^{n_{t-1}} q^{-\left({ }^{n_{t-1}+1}\right)}\left(1-q^{n_{t-1}-\chi(t>m) n_{t-m}}\right) .
$$

This corresponds to $k_{1}=0$, and the sum on $n_{t-1}$ is then

$$
\sum_{n_{t-1}=n_{t-2}}^{n_{t+2}} \frac{(-1)^{n_{t-1}} q^{\left(n_{t-1}\right)-n_{t-2} n_{t-1}}\left(1-q^{n_{t-1}-\chi(t>m) n_{t-m}}\right)}{(q)_{n_{t+2}-n_{t-1}}(q)_{n_{t-1}-n_{t-2}}} .
$$

If $n_{t-2}=n_{t+2}$ then we collapse the sum again and obtain $k_{2}=0$, continuing in this way until $n_{t+a}>n_{t-a}$, and then applying (3.17) and arguing as usual. Note that if $n_{t+m}=n_{t-m}$ then the sum vanishes, so we have $k_{m} \geq 1$.

Using the expression for $C_{n}^{(t, m)}(q)$, we are now prepared to generalize Definition 1.1.

Definition 5.4. The generalized $U$-function $U_{t}^{(m)}(x ; q)$ is defined by

$$
\begin{aligned}
U_{t}^{(m)}(x ; q) & :=\sum_{n=0}^{\infty} C_{n}^{(t, m)}(q)(-x q)_{n}\left(-x^{-1} q\right)_{n} \\
= & q^{-t} \sum_{\substack{k_{t} \geq \cdots \geq k_{1} \geq 0 \\
k_{m} \geq 1}}(-x q)_{k_{t}-1}\left(-x^{-1} q\right)_{k_{t}-1} q^{k_{t}} \\
& \times \prod_{i=1}^{t-1} q^{k_{i}^{2}}\left[\begin{array}{c}
k_{i+1}-k_{i}-i+\sum_{j=1}^{i}\left(2 k_{j}+\chi(m>j)\right) \\
k_{i+1}-k_{i}
\end{array}\right] .
\end{aligned}
$$

By construction, $U_{t}^{(m)}(-1 ; q)$ is dual to $F_{t}^{(m)}(q)$ as follows. 
Theorem 5.5 .

$$
F_{t}^{(m)}\left(\zeta_{N}^{-1}\right)=U_{t}^{(m)}\left(-1 ; \zeta_{N}\right)
$$

Proof. We have $J_{N}^{(t, m)}(q)=U_{t}^{(m)}\left(-q^{N} ; q\right)$ from (5.9); thus, we get

$$
J_{N}^{(t, m)}\left(\zeta_{N}\right)=U_{t}^{(m)}\left(-1 ; \zeta_{N}\right)
$$

With the help of (5.7), we get (5.20).

We end this section with the Hecke-type formula for $U_{t}^{(m)}(x ; q)$. These follow just as those for $U_{t}(x ; q)$ in Theorem 1.3, using the Bailey pair in Proposition 5.2 in place of the Bailey pair in Proposition 3.1.

\section{Theorem 5.6.}

$$
\begin{aligned}
& U_{t}^{(m)}(-x ; q)=-q^{-\frac{t}{2}-\frac{m}{2}+\frac{3}{8}} \frac{(x q)_{\infty}(q / x)_{\infty}}{(q)_{\infty}^{2}}
\end{aligned}
$$

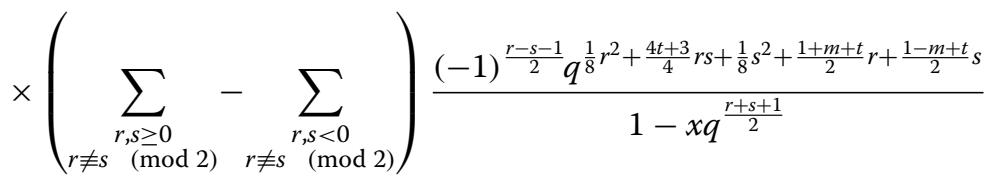

$$
\begin{aligned}
& =-q^{-\frac{t}{2}-\frac{m}{2}+\frac{3}{8}} \frac{(x q)_{\infty}(q / x)_{\infty}}{(q)_{\infty}^{2}} \\
& \times\left(\sum_{\substack{r, s, u \geq 0 \\
r \neq s(\bmod 2)}}+\sum_{\substack{r \neq s, s, u<0 \\
r \neq s(\bmod 2)}}\right)(-1)^{\frac{r-s-1}{2}} x^{u} q^{\frac{1}{8}} r^{2}+\frac{4 t+3}{4} r s+\frac{1}{8} s^{2}+\frac{1+m+t}{2} r+\frac{1-m+t}{2} s+u^{\frac{r+s+1}{2}} .
\end{aligned}
$$

\section{Concluding remarks}

In this paper, we have studied a family of quantum modular forms $U_{t}(-1 ; q)$ based on the colored Jones polynomial for the torus knot $T_{(2,2 t+1)}$. We have extended the duality between $U_{1}(-1 ; q)$ and $F_{1}(q)$ to general $t$ and determined a Hecke-type expansion for $U_{t}(x ; q)$. We have further generalized these results to the vector-valued setting.

We close with two remarks.

First, in $[4,9]$ the modular transformation formula was given for $U_{1}(-1 ; q)$ for a generic $q$, based on an expression for $U_{1}(x ; q)$ in terms of the Appell-Lerch series in [4]. The expression in terms of the Appell-Lerch series also shows that $U_{1}(x ; q)$ is a mixed mockmodular form for generic roots of unity $x \neq-1$ and a mock theta function when $x= \pm i$. Similar results may hold in the general case, and it is to be hoped that the Hecke series expansions established in this paper will turn out to be useful for determining modular transformation formulae for $U_{t}^{(m)}(x ; q)$ for a generic $q$ and for $x$ a root of unity. For now, we only know that by Theorem $5.5, U_{t}^{(m)}(-1 ; q)$ fulfills (5.5) when $q$ is a root of unity.

Second, both $U_{1}(x ; q)$ and $F_{1}(q)$ are interesting combinatorial generating functions. The two-variable function $U_{1}(x ; q)$ can be interpreted in terms of strongly unimodal sequences and their ranks [7,9], while $F_{1}(1-q)$ is the generating function for certain linearized chord diagrams [29] (as well as a number of other objects - see A022493 of [28] for an overview with references.) Moreover, there are many nice congruences for the coefficients of $F_{1}(1-q)$ [5] and $U_{1}(1 ; q)$ [9]. The generalized $U_{t}^{(m)}(x ; q)$ and $F_{t}^{(m)}(1-q)$ may have interesting combinatorial interpretations and congruence properties as well. 


\section{Appendix}

\section{A Examples: $\boldsymbol{t}=\mathbf{2}$ and $\mathbf{3}$}

Here, we record the set of generalized Kontsevich-Zagier series $F_{t}^{(m)}(q)$ and generalized $U$-functions $U_{t}^{(m)}(x ; q)$ for $t=2$ and 3 .

When $t=2$, the set of generalized Kontsevich-Zagier series is

$$
\begin{aligned}
& F_{2}^{(1)}(q)=q^{2} \sum_{n=0}^{\infty}(q)_{n} \sum_{k=0}^{n} q^{k(k+1)}\left[\begin{array}{l}
n \\
k
\end{array}\right]_{q} \\
& F_{2}^{(2)}(q)=q^{2} \sum_{n=1}^{\infty}(q)_{n-1} \sum_{k=0}^{n} q^{k^{2}}\left[\begin{array}{l}
n \\
k
\end{array}\right]_{q} .
\end{aligned}
$$

The dual $U$-functions, satisfying $F_{2}^{(m)}\left(\zeta_{N}^{-1}\right)=U_{2}^{(m)}\left(-1 ; \zeta_{N}\right)$, are given by

$$
\begin{aligned}
U_{2}^{(1)}(x ; q) & =\sum_{n=0}^{\infty}(-x q)_{n}\left(-x^{-1} q\right)_{n} q^{n-1} \sum_{k=1}^{n+1} q^{k^{2}}\left[\begin{array}{c}
n+k \\
2 k-1
\end{array}\right]_{q} \\
& =1+q+\left(x+2+x^{-1}\right) q^{2}+\left(2 x+3+2 x^{-1}\right) q^{3}+\left(3 x+6+3 x^{-1}\right) q^{4}+\cdots \\
U_{2}^{(2)}(x ; q)= & \sum_{n=0}^{\infty}(-x q)_{n}\left(-x^{-1} q\right)_{n} q^{n-1} \sum_{k=0}^{n+1} q^{k^{2}}\left[\begin{array}{c}
n+k+1 \\
2 k
\end{array}\right]_{q} \\
= & q^{-1}+2+\left(x+2+x^{-1}\right) q+\left(2 x+4+2 x^{-1}\right) q^{2}+\left(4 x+6+4 x^{-1}\right) q^{3}+\cdots
\end{aligned}
$$

When $t=3$, the set of generalized Kontsevich-Zagier series is

$$
\begin{aligned}
& F_{3}^{(1)}(q)=q^{3} \sum_{n=0}^{\infty}(q)_{n} \sum_{k=0}^{n} q^{k(k+1)}\left[\begin{array}{l}
n \\
k
\end{array}\right]_{q} \sum_{j=0}^{k} q^{j(j+1)}\left[\begin{array}{l}
k \\
j
\end{array}\right]_{q}, \\
& F_{3}^{(2)}(q)=q^{3} \sum_{n=0}^{\infty}(q)_{n} \sum_{k=1}^{n+1} q^{k(k-1)}\left[\begin{array}{c}
n \\
k-1
\end{array}\right]_{q} \sum_{j=0}^{k} q^{j^{2}}\left[\begin{array}{l}
k \\
j
\end{array}\right]_{q}, \\
& F_{3}^{(3)}(q) \quad=q^{3} \sum_{n=1}^{\infty}(q)_{n-1} \sum_{k=0}^{n} q^{k^{2}}\left[\begin{array}{l}
n \\
k
\end{array}\right]_{q} \sum_{j=0}^{k} q^{j^{2}}\left[\begin{array}{l}
k \\
j
\end{array}\right]_{q} .
\end{aligned}
$$

The dual $U$-functions, satisfying $F_{3}^{(m)}\left(\zeta_{N}^{-1}\right)=U_{3}^{(m)}\left(-1 ; \zeta_{N}\right)$, are given by

$$
\begin{aligned}
U_{3}^{(1)}(x ; q) & =\sum_{n=0}^{\infty}(-x q)_{n}\left(-x^{-1} q\right)_{n} q^{n-2} \sum_{k=1}^{n+1} q^{k^{2}}\left[\begin{array}{c}
n+k-1 \\
2 k-1
\end{array}\right]_{q} \sum_{j=1}^{k} q^{j^{2}}\left[\begin{array}{c}
n+k+2 j-1 \\
2 k+2 j-2
\end{array}\right]_{q} \\
& =1+q+\left(x+2+x^{-1}\right) q^{2}+\left(2 x+4+2 x^{-1}\right) q^{3}+\left(4 x+7+4 x^{-1}\right) q^{4}+\cdots \\
U_{3}^{(2)}(x ; q)= & \sum_{n=0}^{\infty}(-x q)_{n}\left(-x^{-1} q\right)_{n} q^{n-2} \sum_{k=1}^{n+1} q^{k^{2}}\left[\begin{array}{c}
n+k \\
2 k
\end{array}\right]_{q} \sum_{j=0}^{k} q^{j^{2}}\left[\begin{array}{c}
n+k+2 j \\
2 k+2 j-1
\end{array}\right]_{q} \\
= & q^{-1}+2+\left(x+3+x^{-1}\right) q+\left(3 x+5+3 x^{-1}\right) q^{2}+\left(5 x+10+5 x^{-1}\right) q^{3}+\cdots \\
U_{3}^{(3)}(x ; q)= & \sum_{n=0}^{\infty}(-x q)_{n}\left(-x^{-1} q\right)_{n} q^{n-2} \sum_{k=0}^{n+1} q^{k^{2}}\left[\begin{array}{c}
n+k \\
2 k
\end{array}\right]_{q} \sum_{j=0}^{k} q^{j^{2}}\left[\begin{array}{c}
n+k+2 j+1 \\
2 k+2 j
\end{array}\right]_{q} \\
= & q^{-2}+2 q^{-1}+\left(x+3+x^{-1}\right)+\left(2 x+5+2 x^{-1}\right) q+\left(5 x+8+5 x^{-1}\right) q^{2}+\cdots
\end{aligned}
$$




\section{Competing interests}

The authors declare that there are no competing interests.

\section{Authors' contributions}

Both $\mathrm{KH}$ and $\mathrm{JL}$ carried out the research and wrote the manuscript. Both authors read and approved the final manuscript.

\section{Acknowledgements}

KH thanks S. Zwegers for discussions. He also thanks the organizers of 'Lectures on $q$-Series and Modular Forms' (KIAS, July 2013), 'Modular Functions and Quadratic Forms - number theoretic delights' (Osaka University, December 2013), 'Low Dimensional Topology and Number Theory' (MFO, August 2014). The work of KH is supported in part by JSPS KAKENHI Grant Number 23340115, 24654041, 26400079

Received: 28 September 2014 Accepted: 28 October 2014

Published online: 29 January 2015

\section{References}

1. Andrews GE. An analytic generalization of the Rogers-Ramanujan identities for odd moduli. Proc. Natl. Acad. Sci. USA. 1974;71:4082-4085.

2. Andrews GE. Multiple series Rogers-Ramanujan type identities. Pacific J. Math. 1984;114:267-283.

3. Andrews GE. Bailey chains and generalized Lambert series: I. Four identities of Ramanujan. Illinois J. Math. 1992;36: $251-274$.

4. Andrews GE, Rhoades RC, Zwegers SP. Modularity of the concave composition generating function. Algebra Number Theory. 2013;7:2103-2139.

5. Andrews GE, Sellers JA. Congruences for the Fishburn numbers. J. Number Theory. to appear.

6. Bringmann K, Creutzig T, Rolen L. Negative index Jacobi forms and quantum modular forms. Res. Math. Sci. to appear. arXiv:1401.7189 [math.NT].

7. Bringmann K, Folsom A, Rhoades R. Unimodal sequences and "strange" functions: a family of quantum modular forms. Pacific J. Math. to appear.

8. Bringmann K, Rolen L. Half-integral weight Eichler integrals and quantum modular forms. preprint, arXiv:1409.3781 [math.NT]

9. Bryson J, Ono K, Pitman S, Rhoades RC. Unimodal sequences and quantum and mock modular forms. Proc. Natl. Acad. Sci. 2012;109:16063-16067.

10. Choi D, Lim S, Rhoades RC. Mock modular forms and quantum modular forms 2014. preprint.

11. Folsom A, Ono K, Rhoades RC. Mock theta functions and quantum modular forms. Forum Math. Pi. $2013 ; 1: 27$.

12. Habiro K. On the colored Jones polynomial of some simple links. RIMS Kokyuroku. 2000;1172:34-43.

13. Habiro K. A unified Witten-Reshetikhin-Turaev invariant for integral homology sphere. Invent. Math. 2008;171:1-81. arXiv:math/0605314.

14. Hikami K. Difference equation of the colored Jones polynomial for the torus knot. Int. J. Math. 2004;15:959-965. arXiv:math/0403224.

15. Hikami K. $q$-series and L-functions related to half-derivatives of the Andrews-Gordon identity. Ramanujan J 2006;1 1: 175-197. arXiv:math/0303250.

16. Hikami K. Hecke type formula for unified Witten-Reshetikhin-Turaev invariant as higher order mock theta functions. Int. Math. Res. Not. IMRN. 2007;2007:rnm022-32.

17. Hikami K, Kirillov AN. Torus knot and minimal model. Phys. Lett. B. 2003;575:343-348. arXiv:hep-th/0308152

18. Hikami K, Kirillov AN. Hypergeometric generating function of L-function, Slater's identities, and quantum knot invariant. Algebra i Analiz. 2005;17:190-208. arXiv:math-ph/0406042.

19. Lawrence R, Zagier D. Modular forms and quantum invariants of 3-manifolds. Asian J. Math. 1999;3:93-107.

20. Le TTQ. Quantum invariants of 3-manifolds: integrality, splitting, and perturbative expansion. Topology Appl. 2003;127:125-152. arXiv:math/0004099.

21. Lickorish WBR. An Introduction to Knot Theory, vol. 175 of Graduate Texts in Mathematics. New York: Springer; 1997.

22. Lovejoy J. Ramanujan-type partial theta identities and conjugate Bailey pairs. Ramanujan J 2012;29:51-67.

23. Lovejoy J. Bailey pairs and indefinite quadratic forms. J. Math. Anal. Appl. 2014;410:1002-1013.

24. Masbaum G. Skein-theoretical derivation of some formulas of Habiro. Algebraic Geometric Topology. 2003;3: 537-556.

25. Morton HR. The coloured Jones function and Alexander polynomial for torus knots. Proc. Cambridge Philos. Soc. 1995; 117:129-135.

26. Ngo TH, Rhoades RC. Integer partitions, probabilities and quantum modular forms 2014. preprint.

27. Rosso M, Jones V. On the invariants of torus knots derived from quantum groups. J. Knot Theory Ramifications. 1993;2:97-112.

28. Sloane NJA. The On-Line Encyclopedia of Integers Sequences. http://oeis.org. Accessed on 27/11/2014

29. Zagier D. Vassiliev invariants and a strange identity related to the Dedekind eta-function. Topology. 2001;40:945-960.

30. Zagier D. Quantum modular forms In: Blanchard E, Ellwood D, Khalkhali M, Marcolli M, Moscovici H, Popa S, editors. Quanta of Maths, vol. 11 of Clay Mathematics Proceedings. Providence: Amer. Math. Soc.; 2010. p. 659-675. 